\title{
Study on Reliability Optimization Problem of Computer Network
}

\author{
Liang Lei \\ Qingdao Hospitality Management Vocational And Technical College \\ leong1979@163.com
}

\begin{abstract}
With the rapid development of computer network, the problem of reliability of computer network is becoming more and more attention of builders, users and the network designer. The reliability of computer network has become a key technical indicators to measure the comprehensive performance of computer network. Aiming at a single known computer network, applying to established the computer network link cost model and the computer network reliability model, and carries on the simulation using the genetic algorithm intelligent algorithm; comprehensive evaluation for a variety of known different computer network reliability index system, and we have given the evaluation results. The simulation results show that, the established computer network link cost model and the computer network reliability model in the thesis are suitable, model algorithm and programming language is effective, it has the practical application significance which the paper proposed the computer network reliability optimization design concrete plan.
\end{abstract}

Keywords: Computer Network; Reliability; Genetic Algorithm; Optimization

\section{Introduction}

Reliability problems produced in the 1920s. At that time, people just have a preliminary understanding that the problem of reliability. The Second World War, the unreliability problems of weapons and equipment, the Warring States bring the lesson of blood. The successful experience of weapon equipment reliable and unreliable brought from failures, let people gradually deepened on the reliability of the importance of the problem. Therefore, the reliability problem is the people in the social practice process, along with the need of objective situation arising[1]. The birth and development of it is the inevitable trend of social development, development of the science and technology, especially the development of electronic technology is closely connected with. After more than half a century of development, the problem of reliability has become a new cross a comprehensive science involves is very extensive.

Our research of reliability problems start and development and the same to international, and is originated from the study of electronic products. In twentieth Century, the late 50's and early 60's, our country has done some research work on the reliability investigation and application environment, and set up a specialized research institutions, on the failure of electronic products is studied tentatively [2]. In twentieth Century, the late 80 's and early 90 's. Science and technology as the guide, quality as the main line. Under the guidance of the principle, set off a new upsurge in the study of reliability problem of our country, and made great achievements. Reliability work from scratch, quantity and quality of scientific research personnel gradually improve the reliability, the reliability level of products is also rising.

The related concept of the reliability of computer network is appeared in the 1970s, because of the rapid development of communication technology, optical fiber 
technology, computer network technology, making the function of network increase, promoting research on computer network reliability issues continue to depth direction of development. With the acceleration of information society, not only the user of the computer network on the increase, and the connection of regional and network connection of the computer network scale is rapidly expanding. As an important field of computer network is widely used in enterprises and institutions, bank, traffic, communication, industry, national defense, the complexity of the computer network of powerful or not and its structure proportion[3]. The user dependence on computer network more big, to the requirements of computer network becomes high, namely: the accurate, rapid, safe, convenient and reliable. Under the support of computer network system need to complete the desired functionality in a large number of hardware and software, in certain circumstances, sometimes it also need the participation of the people. Once the computer network system failure, it will be to produce economic, political, cultural, health, environment and great social influence and damage, and even endanger the social stability and national security [4]. Thus, we can see the importance of research questions the reliability of computer network and the urgency to study the reliability of computer network has very important theoretical significance and practical value.

With the usage of computer network reliability of the network, people are increasingly high requirements. Once the computer network failure, it will be on people's lives, economic, environmental, health, and social security cause significant even catastrophic effects according to statistics, in the financial field, if the computer network paralysis an hour, it will result in a loss of about $\$ 6000000$, if the computer network paralysis one day, leading to a commercial bank on the verge of bankruptcy. In real life, the computer network fault exists in a large amount, the related cases are: 2005 December Taiwan Strait earthquake led to a number of international submarine communications cable is damaged, so that the whole Asia Pacific area Internet service almost paralyzed, Chinese mainland to Taiwan area, American, Europe and other direction communication line affected by this are many interrupt; 2008 April to July Twitter service, fault occurs repeatedly, cause the interruption of the network, making many users give up Twitter service, ran to the Friendfeed and other competitors, resulting in a large number of users to reduce [5]; 2008 June 17 days, most of the time the Amazon site in America working day interrupt service, makes millions of users are forced to go back to work, the expected loss of $\$ 16000$ per minute; 8 July, 2008, Google online Office application software interrupt service, letting the user's file to stay in the cloud, because the company established online applications such as Google is considered to replace the traditional applications such as Microsoft Office, the service interruption has shaken the confidence of the users for this service, resulting in loss of trust. These computer network failure events and caused huge economic loss, or caused the inconvenient for people to communicate, or caused the user as well as the mistrust of network reliability. Thus, network reliability research has great significance and application value.

\section{Related Research}

Research on network reliability problem of the computer can be traced back as early as $1955 \mathrm{Mr}$. Lee to explore Telecom exchange network, because the network component failure makes Telecom exchange network total transmission capacity greatly reduced, cause the call congestion, resulting in telecommunication exchange Network appear large area paralysis, caused huge economic losses when Mr. Lee will call congestion as link fault network, and for the first time proposed the network 
reliability measure to connectivity as a standard. Research on computer network reliability early mainly concentrated in the field of communication networks.

In foreign countries, the research on computer network reliability problem of the earliest research on Telecom exchange network began Lee. Because the total transmission capacity component failures of computer network makes Telecom exchange network is greatly reduced, resulting in the call congestion, making telecommunication switching network for large area of paralysis[6][7]. Link failure Mr Lee will call congestion definition exchange network for Telecom, and he first proposed the computer network reliability measure to connectivity as a standard. In 1968, it was born in the United States that the world's first computer network, which caused a lot of computer network reliability research. In this period, it is mainly based on the connectivity of the network as the computer network reliability criteria to study. The reliability of computer network connectivity to the standard stipulated in: as long as the computer network link is connected, or between users of computer network communication is connected, or computer network node without fault, the computer network is normal work. International Boecsh, Wilkov, Bgagal famous scholars shows some measure reliability of the network based on connectivity is a standard.

Reliability optimization and design of computer network is proposed firstly by Boecsh, the main issues related to the computer network reliability optimization and improvement of computer network topology. In the course of the discussion of the above two kinds of problem are cannot do without computer network reliability calculation. Calculation of the computer network reliability, Syatnaaarynaa, Baggal and other famous scholars have put forward some specific algorithm.

After 1980s, the rapid development of computer technology makes the computer network has become an important part of the main communication channel and the public life of industrial and commercial, banking, telecommunications, postal, transportation, energy, military and national security. In the background of development of such a large, the index system of the international standard of reliability of computer network with the actual situation of the computer network and constantly changing[8]. For example: the user of the whole network service quality complaints rate; satisfaction of user to network system application; network failure caused by user error rate; fault network system software rate; network transmission! Reliability switching equipment user terminal; reliability; reliability of network links; Web server failure rate; connectivity rate of the whole network; average failure rate per 1000 hours of network; network fault average repair time (in minutes); the network average failure free interval of time (in hours); recognition of the user on the network organization and management system; specific targets users of network security authentication system of recognition and so many evaluation is gradually applied in the computer network reliability calculation and the computer network reliability index system.

Study on the reliability of computer network is increasingly deepening, and constantly open up new field potential. This phenomenon strongly suggest that, on the computer network reliability will gain greater impetus, its scope of application will be more expanded, in practical applications will achieve more fruitful results.

In China, the earliest start of reliability engineering, started in the 1960s, it was put forward by Chinese famous scientist Tsien Hsueshen, and first used in aerospace and military field. To enter in twentieth Century after 70 years, with the extensive application of computer network, researchers gradually began to research the reliability of computer network. At the same time, the emergence of many new computer network reliability algorithm, in the development of the computer network on the theory also has great breakthrough. The domestic scholars and experts have 
published a large number of reliability of computer network article, most IEEE.Trnas.Rel and Miocr\&Rel published in two journals[9]. The famous scholar Zhang Gongxu put forward the concept and algorithm of communication network cut set in 1956, and was applied to the analysis and Study on the reliability of computer network.

In the early research on computer network reliability, it is mainly based on computer network connectlno and cohesion as the measure of the reliability of computer network. For the actual computer network communication service, must complete certain, sometimes even when the computer network is connected, but the volume of business computer networks do not necessarily meet the expectation. So this is also studies the question the reliability of computer network is one of the barriers.

Since 1990s, all over the domestic application of computer network tide is advancing wave upon wave, from a few computer network to a few kilometers and thousands of kilometers wide area network in an office, office building, to realize sharing of hardware, software and data resources. However, when the computer network malfunction, bring their own losses could reach irreparable point. According to the data show: in a large domestic enterprises, an annual average of computer network reliability problems caused by the loss has reached millions of dollars, while the internal computer network failure rate of about two times a month, average every day a computer network can not run. In the field of finance, computer network paralysis of one hour each, will result in a loss of $\$ 6000000$; if the computer network paralysis a day, can make an investment in commercial banks failed completely[10]. These amazing case, let the computer network planners, designers, builders and users can't ignore the reliability problems of computer network, to improve the reliability of computer network, it should reduce the failure rate, the importance of design and optimization of the reliability of computer network.

At present, the domestic research on the reliability problems of computer network hardware facilities has made significant progress, and gradually formed a relatively complete theoretical system, to evaluate research focus is gradually shifted to the computer network reliability of network management software and computer network reliability index system. Domestic research work the computer network reliability than international nearly 20 years later, in the ideological understanding, organization management, human, material and financial resources, and many other factors, the research work on the reliability of computer network system in less. Problems in the design of computer network reliability optimization is one of the most popular field of study. Therefore, the study of related theory on the reliability of computer network and the technology is feasible, and it has theoretical and practical value.

\section{Proposed Scheme}

\subsection{The Define of Computer Network Reliability and Reliability}

The concept of reliability of computer network as a system engineering science, after more than half a century of development, has formed a relatively complete, sound system. Domestic and foreign scholars to measure the reliability of computer network are classified into four categories: computer network connectivity, computer network survivability, computer network, computer network components damage resistance in multimode work effectiveness. If the normal work of the computer network, the basic nodes and components in the network must provide a 
reliable link to each user terminal. Therefore, the connectivity of computer network reliability in the relevant research in the field of the most widely used. Connectivity of computer network generally use the computer network reliability to measure.

(1) The reliability of computer network. The computer network on the terms and conditions (mode of operation, repair mode, load conditions, temperature, humidity, radiation, etc.), the provisions of the time (1000 hours, a quarter, etc.), keeping network connectivity and the ability to meet the communication requirements, called the reliability of computer network. It reflects the ability of computer network topology structure to support the normal operation of the computer network, computer network programming! One of the important parameters of design and operation.

(2) Definitions of reliability of computer network. The computer network on the terms and conditions (mode of operation, repair mode, load conditions, temperature, humidity, radiation, etc.), the provisions of the time (1000 hours a quarter, etc.), network to complete the provisions of the probability function, called the computer network reliability, is ${ }^{R(t)}$, where ${ }^{R(t)=P\{T>t\}}$. The computer network reliability has three types:

(1) 2-terminal reliability, namely in the probability graph, specifying the source Reint: the probability of at least one link between a normal operation and sink $\mathrm{t}$, is

(2) $\lambda$-terminal reliability, namely in the probability graph, which consists of a specified node set in two pairs of nodes between the probability of link, all the normal operation of the, is $\operatorname{Re} l_{\lambda}(G)$.

(3) All terminal reliability, namely in the probability graph, specify any two nodes among households, the probability that the link has the normal operation of the, is $\operatorname{Re} l_{A}(G)$.

From the above definition we can get, when $\lambda=2$ or $\lambda=n, \lambda$-terminal reliability is 2-terminal reliability or all terminal reliability. So the 2-terminal reliability and all terminal reliability can be regarded as a special case of $\lambda$-terminal reliability. In general, we used $\operatorname{Re} l(G)$ to denote the floorboard of the above three kinds of computer network reliability.

\subsection{Computer Network Reliability Model}

In the system engineering of complex systems, many even complex giant systems can be modeled as a network model[11], the network model and further model for reliability problem of graph to solve, such as energy supply network, communication network, transportation network, troops and equipment material support network and so on. The computer network reliability problems can also be modeled as reliability problem of a graph. Computer network model by probability graph $G(V, E)$ to represent, in which nodes $V$ represent the collection of user terminal of the computer network, host or server, edge set $E$ link computer network. Problems of this type generally has the following six assumption:

(1) The computer network is connected, the available mathematical diagram $G(V, E)$ to describe, and between any two nodes in the graph $\mathrm{G}$ is no more than a directly connected link;

(2) Reliability and medium computer network link medium regardless of the length of the;

(3) Computer network node itself is not failure;

(4) Computer network nodes and links of the working state of only two: normal and fault, and the fault occurrence is independent of each other; 
(5) The probability of the normal operation of the computer network nodes and links of the known, the normal state and the computer network node and link is independent of each other in probability and statistics;

(6) Computer network model can also be used to describe the multistate model.

Probability graph model of computer network, is obtained after the normal operation of the state of each edge of the graph and the nodes of the given a certain probability value map. The reliability problem of graph contains two aspects: one is the analysis of the problem, namely, the calculation of the reliability of a given graph; two is a design problem, namely, in the given all the elements, design with maximum reliability diagram[12]. Diagram of the reliability is not convenient to solve, can seek first the failure degree (reliability failure degree $+=1$ ), and then the reliability. The node and link diagrams failure model can be divided into the link failure model, node failure model, node and link failure model of mixing three types, of which. The node and link hybrid failure model. The most commonly used, this article mainly aims at this kind of problem research.

\subsection{Effect of Network Equipment for Network Reliability}

(1) Influence of user equipment on the reliability of computer network. The user terminal is the direct user oriented equipment, it is important for the reliability, and it is also the key to the computer network reliable or not. Routine maintenance in the computer network in the process of operation, the main is to ensure that the user terminal reliability. Ability to interact with the user terminal is higher, the network reliability is also higher. For example, the installation of two pieces of card, connected to the LAN segment different, to a much higher than the reliability of a network card number. The server is the computer network information center and service provider, it usually includes database server, file server, print server, e-mail server, the event server, web server. These sub server is reliable or not directly affects the reliability and efficiency of computer network. Generally speaking, the server error recovery capability and reliability of response time is proportional to the. In improving the reliability of sub server at the same time, we should adopt dual switching system server, namely in the computer network operation system, using two servers, a as the master and the other as a backup machine. Although it would promote the cost of computer network is increased, but the corresponding reliability of computer network is improved with the increase of.

(2) The influence of transmission and switching equipment for the computer network reliability. In the practice of computer network construction, operation, researchers often find:. The problem of computer network fault wiring system caused by the general is the most difficult to find, therefore the cost is often the biggest.. Therefore, we should adopt the standard of communication lines and wiring system. In order to improve the reliability of computer network and meet the need of the future development of the computer network, must consider a certain redundancy and fault tolerance. For the very important and not worry about the cost of construction of the computer network, the wiring is best arranged to double, so that the computer network line failure to timely switch. Computer network hub, a plurality of user terminals connected to the network through together, it can be connected device error and computer network other parts separated, constitute the first line of defense to ensure the reliability of computer network. The hub is a kind of equipment for single point of failure, if its failure, is connected with the user cannot work, the hub of visible computer network plays an important role in improving the reliability of computer network. Therefore, in the design of the computer network, often have to consider adopting effective measures to ensure its 
reliability. Switches used to connect several independent LAN, and data packet filtering a network interconnection equipment in its, can be used to solve the bottleneck problem of insufficient bandwidth and network exchange, meet between the user terminal and server interaction needs, improving the performance of the entire network. The router is one of the interconnection device network layer, use it not only can realize interconnection with the type of local area network, but also can achieve LAN

3.4 The design principles of computer network reliability

In the engineering practice of computer network design and construction, scientific research personnel to sum up a lot of specific design experience and the principle, optimization design of the reliability of the computer network has played a good role in standardizing and guiding. In the construction of computer network should follow the following principles:

(1) According to international standards, the use of computer network open system structure, effective interconnection support heterogeneous systems and different equipment, with strong expansion and upgrade ability;

(2) The advanced and mature, practical, universal combination, selection of advanced computer network technology and mature, the choice of practical and general computer network topology;

(3) Computer network must have a strong ability of interconnection, can support a variety of communication protocols;

(4) The safety, the reliability of computer network to high, with strong redundancy and fault tolerant ability, to strengthen the security of data, ensure the normal operation of the whole network system, the system should select the server and computer network product has strong redundancy fault-tolerant ability;

(5) The computer network management, should choose the network equipment network software and support SNMP and CMPI management of the advanced;

(6) Computer network link better medium, backbone network with enough bandwidth, the network has faster response speed;

(7) The protection of existing network investment, make full use of the existing resources of a computer network, a reasonable allocation of existing hardware facilities, network cabling, the mature network operating system software and network application software;

(8) The reliability of computer network performance to price ratio should be as high as possible.

\subsection{The Optimization Model of Computer Network Reliability}

Theoretically, to enhance the reliability of computer network related parts and attach the appropriate redundant components are two main ways of improving the reliability of computer network. To meet the expected function in the computer network under the premise, using the redundant technology (increasing the standby link number) on the one hand can improve the reliability of local fragments of the computer network; on the other hand also improves the computer network construction cost. Because each computer network link are reliability and cost, the number of the computer in the network link to less, accordingly, the higher the reliability of computer network. This paper gives the computer network reliability optimization model, first determine the reliability matrix computer network link is as follows: 


$$
R_{0}=\left[\begin{array}{cccc}
r_{11} & r_{12} & \ldots & r_{1 n} \\
r_{21} & r_{22} & \ldots & r_{2 n} \\
\ldots & \ldots & \ldots & \ldots \\
r_{m 1} & r_{m 2} & \ldots & r_{m n}
\end{array}\right]
$$

Where $R_{0}$ is the computer network reliability matrix; $r_{i j}$ is computer network node $\mathrm{i}$, link reliability of $\mathrm{J}$ between the. $1 \leq i \leq m, 1 \leq j \leq n$

Secondly, in $G=(N, L)$, May be a subset $L^{\prime}$ of $L$ in the normal state, $L^{\prime} \subseteq L$, All nodes are in the normal state of the reliability of $\prod P\left(n_{i}\right)$, Therefore, the computer network reliability optimization model can be diescribed as abstract : the optimum number of computer network links identified, Maximizing $\operatorname{Re} l(G)$, and $C \leq C_{0}$. Computer network reliability optimization model to establish the following

$$
\begin{aligned}
& \operatorname{Re} l(G)=\left|\sum_{\Omega}\right| \prod_{i \in L} P\left(l_{i}\right)|| \prod_{i \in\left(L / L^{\prime}\right)}\left(1-P\left(l_{i}\right)\right) \mid \bullet \prod_{i=1}^{n} P\left(n_{i}\right) \\
& \operatorname{Max} \operatorname{Re} l(G) \\
& \operatorname{Min} C=\sum_{i=1}^{N} \sum_{j=1}^{i} c_{i j} g_{i j} \\
& \text { s.t } \quad D_{i} a_{i j} \leq \alpha(i, j=1, \ldots, N) \\
& \quad \sum_{j=1, j \neq 1} g_{i j} \geq \beta(i=1, \ldots, N)
\end{aligned}
$$

Where: $\Omega$ - A collection of all of the normal state of the computer network ;

$n_{i}$-The i -node computer network;

$l_{i}$-I- link computer networks ;

$P\left(l_{i}\right)$-Computer network i- link reliability.

Because the computer network reliability optimization model established in this paper is non-linear state, a smart algorithm to solve it is feasible and effective. In this paper, the genetic algorithm to solve the optimization model of computer network reliability.

\subsection{Reliability Optimization Design based on Genetic Algorithms}

Genetic algorithm (Gneetic Algorhitm, referred to as GA) is an effective method to solve the optimization problem. The first it is inspired by the J.Holand in 1975 by evolutionism, and puts forward the. GA is based on the "survival of the fittest" is a highly parallel, random and adaptive optimization algorithm, it shows the solution of the problem as "survival of the fittest chromosomes during", through the "chromosome" group of generations of evolution, including reproduction, crossover and mutation, eventually converge to the "best adapted to their environment" the individual, thus obtains the optimal solution of the problem or satisfactory solution. At present, with the development of computer technology, GA has been paid more and more attention, and in machine learning, pattern recognition, image processing, 
neural network, optimal control, combinatorial optimization, VLSI design, genetics and other fields have been successfully applied.

The genetic algorithm provides a general framework to solve the optimization problems of complex system, and it is not dependent on the type of the field problem. The need for a practical application of optimization calculation, the general genetic algorithm according to the following steps to construct the solution of the problem.

1) Define the decision variables and various constraint conditions, that determine the individual phenotype $\mathrm{X}$ and the solution of the problem space;

2) Optimization model is built, which means to obtain the type of objective function (is the minimum values of the maximum value for the objective function or for the objective function) or quantitative method and its mathematical description form;

3) Determine the representation of chromosome coding method of feasible solution, that is to determine the genotype of $\mathrm{X}$ individuals and genetic algorithm search space;

4) determine the decoding method, and identified by the individual genotype correspondence or convert $\mathrm{X}$ to individual phenotype $\mathrm{x}$ method;

5) Determine the individual to adapt to the quantitative evaluation methods of the degree of which is determined by the value of the objective function $\mathrm{f}(\mathrm{x})$ to the individual fitness degree $\mathrm{F}(\mathrm{X})$ conversion rules;

6) Design of genetic operator, which means to obtain the specific operation method selection operation, crossover operation and mutation operation of genetic operator;

7) Determine the parameters for the operation of the genetic algorithm, which determine the initial population size evolution of Pop-size algebra $\mathrm{T}$, genetic algorithm crossover probability and mutation probability parameters of a few a few.

Design and coding method, the feasible solution in genetic algorithms genetic operators are two main issues that need to be considered when the tectonic genetic algorithm, but also the design of genetic operator of two key steps "specific genetic algorithm need when the coding method using different operations on different optimization problem, the specific problems they are solving closely related, so the understanding degree of the solution is the key to the successful application of genetic algorithm.

(1) Gene expression. This paper uses binary coding method, to determine the expression of $\mathrm{N}$ gene with computer network node, as shown in table 1.

Table 1. Computer Network Node Gene Table Expression

\begin{tabular}{|l|l|l|l|}
\hline$N_{1}$ & $g_{11}$ & $\ldots$ & $g_{1 n}$ \\
\hline$N_{2}$ & $g_{21}$ & $\ldots$ & $g_{2 n}$ \\
\hline$\ldots$ & $\ldots$ & $\ldots$ & $\ldots$ \\
\hline
\end{tabular}

(2) The selection of fitness function. In order to effectively prevent the cheating phenomenon in genetic algorithms to occur, will the population cost value, are arranged according to the numerical size, cost numerical minimum individual sort code is set to 1 , the cost of numerical maximum individual sort code is set to Pop-size, the design of fitness function is as follows:

$$
f(x)=(x-1) /(\text { pop }- \text { size }-1)
$$

Where: $\mathrm{x}$-the position of individual in the cost sequencing; 


$$
\text { Pop-size -a population size. }(1 \leq x \leq \text { Pop - size })
$$

(3) The evolutionary computation (option). This paper by the way of "runner selection", the basic idea is that each gene selection probability proportional to its fitness. For fitness for human gene, the choice probability ruler is calculated according to the following formula.

$$
P_{k}=f_{k} / \sum_{j=1}^{p o p-s i z e} f_{i}
$$

(4) The genetic arithmetic. Computer network node on the expression of gene, pling the method of random node cross, that is to generate a random number from $[1, N]$, determining the gene crossover locations, each can only cross on a node location. Under normal circumstances, the crossover rate $P_{c} \in(0.01,0.1)$.

(5) Adjusting the algorithm steps. When reflect the original computer network topology structure of the computer network node gene expression can not accurately, according to the following steps to achieve the adjustment algorithm:

Step1: For all gene expression one by one judge connected network structure;

Step2: If $g_{i j}=1$, then the implementation of the original crossover, mutation operation;

Step3: If $g_{i j}=0$, then $g_{i j}=1$.

Step4: If $g_{i j}=1$, then $g_{i j}=0$.

Step5: If the Step4 can not realize, in turn, step Step1.

(6) The termination conditions of genetic algorithm. Convergence of GA shows that the limit properties of probability convergence of $\mathrm{L}$, the termination condition is the most commonly used previously set a maximum number of iterations of genetic operation, or in the evolution degree of population is the current generation of the maximum fitness value to population control and population average fitness proportional relationship.

\section{Experimental Results and Analysis}

This paper cite optimization simulation example calculation of a computer network reliability. Computer network link cost model to see the model, points $\mathrm{N}=6$ node computer network, computer network node reliability constraint constant $\alpha=2$ ,$\beta=2$, the number of iterations of genetic operation is 100 times. The cost matrix of computer network link $C_{0}$ and computer network reliability matrix $R_{0}$ ,respectively as follows.

$$
C_{0}=\left[\begin{array}{cccc}
0 & 5 & 8 & 12 \\
5 & 0 & 9 & 4 \\
8 & 9 & 0 & 5 \\
7 & 8 & 11 & 3
\end{array}\right]
$$




$$
R_{0}=\left[\begin{array}{cccc}
0 & 0.98 & 0.95 & 0.99 \\
0.98 & 0 & 0.97 & 0.998 \\
0.99 & 0.996 & 0.95 & 0.97 \\
0.96 & 0.98 & 0.97 & 0.93
\end{array}\right]
$$

According to the genetic algorithm process and genetic algorithm to tune the algorithm flow, step by step simulation. When the number of iterations of genetic operation is 100 times, the simulation process will stop. Through the simulation, the minimum cost of computer network link is 45 , and ensure the maximum value of the computer network reliability is 0.885 . Computer network link cost simulation curve and the computer network reliability simulation curves are shown in figure 1 and figure 2 .

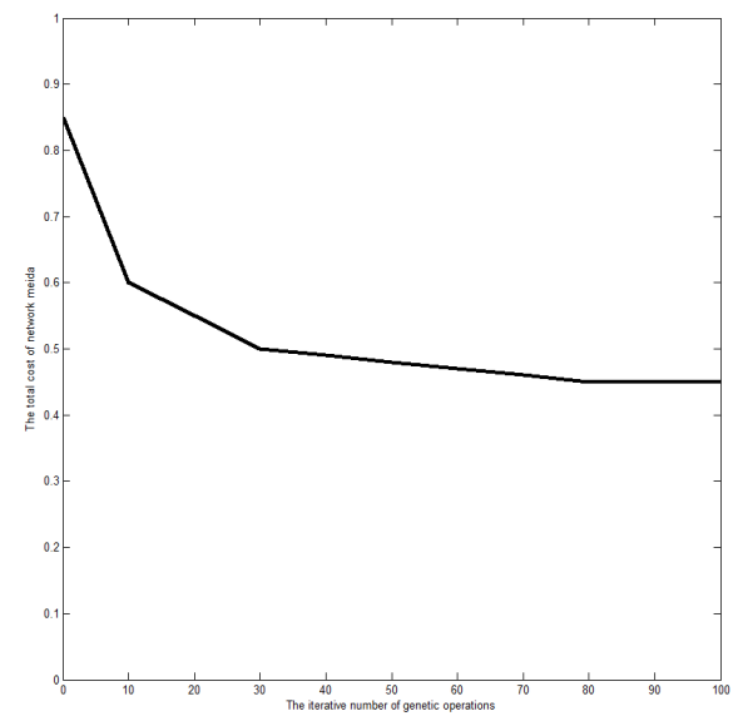

Figure 1. The Simulation Graphs of the Cost of Computer Network Link

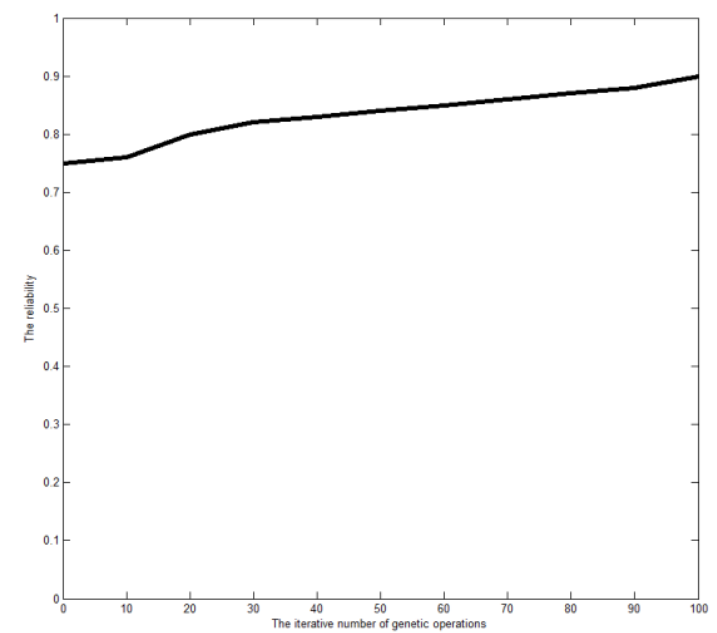

Figure 2. The Simulation Graphs of the Reliability of Computer Network 


\section{Conclusion}

The problem of reliability optimization design of computer network is a complex optimization problem. Genetic algorithm, neural network, fuzzy neural network intelligent algorithm provides a new idea and approach for solving this kind of problem. Based on the analysis of the impact factors of the basis of the reliability of computer network, the computer network link cost model and the computer network reliability model, the application of genetic algorithm to solve the computer network link cost model and the computer network reliability evaluation model, respectively, different kinds of computer network reliability index system. The rapid development of the computer network, increasing factors affecting the reliability of computer network promote hand, on the other hand, put forward higher requirements "therefore, in order to measure the impact of computer network reliability index system of computer network reliability is mentioned in the paper and the factors of computer network reliability index system should be perfected, enriched and improved. The establishment of computer network link cost model and the computer network reliability model should also be considering the randomness of the computer network in the actual operation of the process! Objective function of uncertainty and human factors.

\section{References}

[1] S. T. Y. Lee, K. L. Hicks and E. Hnyilicza, "Transmission expansion by branch-and-bound integer programming with optimal cost-capacity curves, Power Apparatus and Systems, IEEE Transactions, vol. 5, (1974), pp. 1390-1400.

[2] M. G. Rabbat, R. D. Nowak and J. A. Bucklew, "Generalized consensus computation in networked systems with erasure links", Signal Processing Advances in Wireless Communications, IEEE 6th Workshop, IEEE, (2005), pp. 1088-1092.

[3] S. Kartik and C. S. R. Murthy, "Task allocation algorithms for maximizing reliability of distributed computing systems", Computers, IEEE Transactions, vol. 46, no. 6, (1997), pp. 719-724.

[4] B. Dengiz, F. Altiparmak, A. E. Smith, "Efficient optimization of all-terminal reliable networks, using an evolutionary approach", Reliability, IEEE Transactions, vol. 46, no. 1, (1997), pp. 18-26.

[5] T. He, L. Hong and D. Chen D, "Reliable path for virtual endoscopy: ensuring complete examination of human organs", Visualization and Computer Graphics, IEEE Transactions, vol. 7, no. 4, (2001), pp. 333-342.

[6] S. Chowdhury and M. A. Breuer, "Optimum design of IC power/ground nets subject to reliability constraints", Computer-Aided Design of Integrated Circuits and Systems, IEEE Transactions, vol. 7, no. 7, (1988), pp. 787-796.

[7] W. Zhang, J. Tang and C. Wang, "Reliable adaptive multipath provisioning with bandwidth and differential delay constraints", INFOCOM, Proceedings IEEE, IEEE, (2010).

[8] S. Soni, S. Narasimhan and L. J. LeBlanc, "Telecommunication access network design with reliability constraints", Reliability, IEEE Transactions, vol. 53, no. 4, (2004), pp. 532-541.

[9] M. Pedro, E. Monteiro and F. Boavida, "Multi-Perspective Optimization of GEANTInter-domain Traffic", Communication Networks and Services Research, CNSR, Fifth Annual Conference, IEEE, (2007).

[10] Y. Lei, Y. Yuan and J. Zhao, "Model Based Detection and Monitoring of the Intermittent Connections for CAN Networks", (2014).

[11] W. Cai W, X. Jin and Y Zhang Y, "Research on reliability model of large-scale wireless sensor networks", Wireless Communications, Networking and Mobile Computing, WiCOM, International Conference, IEEE, (2006).

[12] J. Xu, "Topological structure and analysis of interconnection networks", Springer Publishing Company, Incorporated, (2010). 


\section{Author}

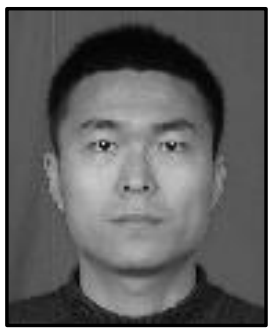

Liang Lei, he received his Master degree in Shandong University of Science and Technology in 2006. Now he is currently working in Qingdao Hospitality Management Vocational And Technical College.

His current research interests on network security and internet of things(IOT). 
International Journal of Security and Its Applications

Vol. 9, No. 4 (2015) 